\title{
Nathalie Mons, Les nouvelles politiques éducatives
}

Pierre-Louis Gauthier

\section{CpenEdition}

\section{Journals}

Édition électronique

URL : http://journals.openedition.org/ries/315

DOI : 10.4000/ries.315

ISSN : 2261-4265

Éditeur

Centre international d'études pédagogiques

Édition imprimée

Date de publication : 1 avril 2008

Pagination : $20-23$

ISBN : 978-2-85420-572-5

ISSN : 1254-4590

Référence électronique

Pierre-Louis Gauthier, « Nathalie Mons, Les nouvelles politiques éducatives », Revue internationale d'éducation de Sèvres [En ligne], 47 | avril 2008, mis en ligne le 29 juin 2011, consulté le 22 septembre 2020. URL : http://journals.openedition.org/ries/315 ; DOI : https://doi.org/10.4000/ries.315

Ce document a été généré automatiquement le 22 septembre 2020.

(c) Tous droits réservés 


\title{
Notes de lecture
}

\section{Nathalie Mons, Les nouvelles politiques éducatives}

\author{
Pierre-Louis Gauthier
}

\section{RÉFÉRENCE}

Nathalie Mons, Les nouvelles politiques éducatives, , PUF, 2007, 202 p. Pierre-Louis Gauthier

1 La crise de l'éducation dans le monde, clairement explicitée par Philip H. Coombs dès $1985^{1}$, n'en finit pas d'alimenter la littérature sur le sujet. L'ouvrage de Nathalie Mons se présente comme une tentative pour mettre un peu de rationalité dans une problématique qui, les incohérences des réformateurs aidant, tourne parfois à la cacophonie.

2 Afin d'identifier les orientations politiques dominant le monde éducatif, l'auteur se propose d'explorer trois pistes: les politiques de décentralisation, les politiques de différenciation de l'école unique, le libre choix de l'école.

3 Nathalie Mons se fixe comme objectif d'évaluer les politiques éducatives et les principales réformes menées en France, à l'aune des comparaisons internationales. Son étude se base sur les données d'Eurydice, de l'OCDE, notamment du programme international pour le suivi des élèves (PISA), ainsi que sur l'abondante production sur le sujet dont les rapports de la cour des comptes, de la direction de l'évaluation de la prospective et de la performance et de l'inspection générale du ministère de l'Éducation nationale. L'ouvrage puise des éléments de comparaison dans les pays développés: Europe, États-Unis, Japon, Australie, Nouvelle-Zélande. En revanche, l'ouvrage n'offre aucune échappée vers les géants indien ou chinois, non plus que vers les autres «dragons » que sont Singapour, Taïwan ou même le Brésil qui connaissent en ce $\mathrm{XXI}^{\mathrm{e}}$ siècle commençant une évolution inouïe de leurs systèmes éducatifs. 
4 Le recours à la comparaison internationale a pour vertu de ramener les phénomènes éducatifs à leur propre échelle même si une part de culture et d'histoire nationales rendent le fait éducatif irréductible à la seule comparaison statistique. L'ouvrage s'ouvre sur un aperçu méthodologique d'autant bien venu qu'il est plus rare dans la littérature universitaire, les méthodes comparatives étant le plus souvent données comme allant de soi. Cependant, on peut se demander s'il était nécessaire de maintenir dans un ouvrage destiné à un public élargi, l'habitude universitaire des références bibliographiques, fort nombreuses et au demeurant récurrentes, qui émaillent le texte au risque d'en alourdir la lecture.

5 Dans le premier volet de l'ouvrage, la décentralisation est déclinée sous ses divers avatars. Nathalie Mons en souligne les ambiguïtés nées du désengagement de l'État, leitmotiv quasi mondial. Elle examine dans ce domaine les mesures prises depuis 1983 qui, laissant la bride sur le cou aux collectivités territoriales, aboutissent à des politiques souvent disparates, faute d'un cadrage national visible. En fait, l'absence d'une culture de l'autonomie en France aboutit à une politique timorée en dépit de la subsidiarité des compétences. Le volet sur la décentralisation, éclairé par la comparaison internationale, met en évidence les formes que peut prendre cette évolution. La typologie fine présentée par Nathalie Mons permet de mettre de l'ordre parmi les conceptions différentes des pays fédéraux ou des pays centralisés et d'échapper à l'opposition simpliste des systèmes. En examinant le détail des compétences, on saisit combien les attributions se complètent sans jamais aboutir à de purs systèmes centralisés ou décentralisés.

6 Le grand intérêt de cette méthode est de mettre en regard les principaux thèmes et le rendement scolaire. Certes, la relation n'est pas simple à établir. Néanmoins, les résultats partiels présentés par l'auteur montrent que les réformes de gouvernance ont des effets limités sur les performances. Dans le cadre d'une décentralisation poussée, on aboutirait, dans bien des cas, à un renforcement de la ségrégation sociale et des inégalités scolaires.

7 Le deuxième volet de l'ouvrage aborde la problématique de l'école « unique ». Ce terme est polysémique en français et il eût mieux valu, pour distinguer la scolarité obligatoire de l'école rurale française, utiliser école de base, fondamentale, moyenne, unifiée, ou par défaut l'anglicisme comprehensive school si le français n'y peut aller, comme aurait pu dire Montaigne. Nathalie Mons parvient à cerner le phénomène de la scolarité longue à travers diverses acceptions internationales. La comparaison à laquelle elle procède concerne plus particulièrement le second degré inférieur. À travers les exemples des États-Unis, du Japon, de la Corée du sud, on voit bien que l'unification de l'école longue n'a jamais été totalement réalisée. L'historique du collège "à la française " permet de rappeler que les réformes se placent dans le temps historicopolitique, ce qui les rend rapidement obsolètes. Plus pertinent est l'examen des politiques concernant le collège qui suivront la loi de 1989. Politiques hasardeuses, hésitantes, générant les disparités géographiques que l'on sait. À la lumière de la comparaison avec les écoles moyennes européennes et notamment scandinaves, Nathalie Mons montre que des voies alternatives étaient possibles, mais que décidément l'imagination n'était plus au pouvoir! L'auteur rappelle la part de responsabilité des politiques et autres groupes de pression. En effet, les décisions successives s'orientent plus vers le «démantèlement » du collège unique initié par De Gaulle, à travers toute une politique de dérogations et d'exceptions jusqu'au récent 
«apprentissage junior» de Gilles de Robien, que vers une refondation d'une école unifiée basée sur l'individualisation des parcours des élèves. Plusieurs modèles s'imposent dans la typologie de l'école obligatoire proposée par Nathalie Mons, de la « séparation » des parcours scolaires à leur intégration.

8 Là aussi, la mise en relation avec les performances scolaires autorise une évaluation de l'efficacité des différents systèmes suivant des indicateurs tels que le niveau général suivant les scores PISA, les inégalités scolaires et les disparités sociales. Les conclusions mettent en évidence, par exemple, le faible niveau généré par les systèmes filiarisés corrélé à des inégalités sociales fortes.

Le troisième thème abordé par Nathalie Mons concerne le libre choix de l'école. L'auteur traite de la sectorisation et des efforts actuels pour assouplir la carte scolaire autant que du « quasi-marché » que représente l'éducation. Dans un contexte libéral de libre concurrence, la désectorisation est évidemment la condition première de la marchandisation de l'éducation. Cette "marketisation" conforme aux lois de la concurrence devait théoriquement maximiser la satisfaction du couple producteur/ consommateur d'éducation. Or les règles du marché appliquées à l'éducation se heurtent au cœur de la scolarité obligatoire nécessairement protégée et réglementée comme tout bien public. En France, le débat est réellement politique avec les récentes déclarations présidentielles sur le libre choix, mais les parents se révèlent peu consommateurs d'école à l'exception des classes aisées qui détiennent l'information sur les établissements. De la typologie établissant les relations statistiques entre le libre choix et les performances, toujours sur la base des données du PISA, on retiendra le peu d'efficacité de ces réseaux scolaires marchandisés: les systèmes éducatifs qui comportent une carte scolaire stricte, sans dérogation, connaissent, semble-t-il, les écarts scolaires et sociaux les plus faibles.

10 En conclusion, Nathalie Mons dégage deux modèles d'école dominants pour le siècle commençant, autour du triptyque : décentralisation, école « unique » filiarisée ou non, libre-choix de l'école. Ces modèles, issus de thèses néolibérales, remettent en cause les relations historiques des États avec leurs systèmes éducatifs. En fait, aucune de ces orientations ne peut être corrélée à une amélioration significative des résultats. Bien des questions se posent au long de cette lecture : ces concepts sont-ils tous du même ordre? Quelle est leur importance relative? Quid d'autres items tels que la formation des enseignants, le niveau de recrutement, le niveau des salaires tellement dissemblables en Europe, la gouvernance des systèmes, la corrélation entre dépenses d'enseignement et performances?

11 La qualité majeure de l'ouvrage de Nathalie Mons est sans doute d'inciter le lecteur à se poser ou se poser de nouveau les questions fondamentales qui devraient fonder toute réflexion sur l'évolution de l'éducation.

\section{NOTES}

1. The World Crisis in Education, Oxford University Press, 1985. 


\section{AUTEURS}

\section{PIERRE-LOUIS GAUTHIER}

Inspecteur d'académie honoraire. 